\title{
Short fiber Munguba (Pseudobombax Munguba) and sprue (Guarea guidonia)
}

\author{
Raimundo Valdan Pereira Lopes ${ }^{1}$ e Eleodoro Rodriguez Hermenegildo ${ }^{1}$ \\ ${ }^{1}$ Universidade Federal do Amazonas - Instituto de Natureza e Cultura-INC/UFAM. Estrada $1^{\circ}$ de Maio - S/N, Colônia. Benjamin \\ Constant- Amazonas- Brasil. CEP: CEP: 69.630-000. Fone: +55(92) 99387 8930, +55(97)991987724 e +55(97)3415-5677.
}

\begin{abstract}
This paper aimed to evaluate the mechanical properties in tensile testing, of polyester composites reinforced by short fibers of Munguba (Pseudobombax Munguba) and Jitó fibers (Guarea guidonia) arranged randomly. The method used was qualitative and quantitative, comparing parameters obtained in tensile mechanical tests of and appearance of the fracture for both types of composites investigated. The results showed that for the investigated composites, the tensile strength increased respectively to the length of the fibers of Munguba (10 mm - 12.16 (MPa) and $15 \mathrm{~mm}-19.91(\mathrm{MPa}))$ and from the Jitó fibers ((10 mm - 18.84 (MPa) and $15 \mathrm{~mm}-. .219 .20(\mathrm{MPa})$ ) Besides, as they remained better aligned, uniform and occupying a larger contact area in the array, the tensile strength also increased. The composites reinforced by fibers of Jitó were the ones who have more satisfactory mechanical performance in tensile test for such process, with respect to manufactured by Munguba fibers.
\end{abstract}

Keywords: Polymeric composites; Munguba fibers (Pseudobombax Munguba); Jitó fibers (Guarea guidonia); Mechanical properties; Tensile test.

\section{Fibras curtas de munguba (pseudobombaxmunguba) e de jitó (guarea guidonia)}

\section{RESUMO}

O artigo em questão objetivou a avaliação das propriedades mecânicas em ensaio de tração de compósitos de poliéster reforçados por fibras curtas dispostas aleatoriamente de Munguba (Pseudobombax munguba) e, os reforçados por fibras de Jitó (Guarea guidonia). O método utilizado foi o quali-quantitativo, confrontando parâmetros obtidos nos ensaios mecânico de tração e aspecto da fratura para os dois tipos de compósitos investigados. Os resultados revelaram que, para os compósitos investigados, a resistência à tração aumentou respectivamente ao comprimento das fibras de Munguba ((10mm - 12,16(MPa) e $15 \mathrm{~mm}$-19,91(MPa)) e de Jitó ((10 mm - 18,84 (MPa) e $15 \mathrm{~mm}$ - 19,08 (MPa)). E, a medida que estas se mantinham melhor alinhadas, uniformes e ocupavam uma área de contato maior na matriz. Sendo os compósitos reforçados por fibras de Jitó, os que apresentaram desempenho mecânico mais satisfatório no ensaio de tração para este tipo processo.

Palavras-chave: Compósitos poliméricos; Fibras de Munguba (Pseudobombax munguba); Fibras de Jitó (Guarea guidonia); Propriedade Mecânica; Ensaio de tração.

\section{INTRODUÇÃO}

Nos últimos anos muitos esforços vêm sendo empregado por parte da indústria na busca por novos materiais com propriedades ecológicas e tecnológicas capazes de atender a novos requisitos tecnológicos e ambientais, em resposta à ineficiência no uso de materiais ditos convencionais e da dependência de matérias-primas provenientes de fontes não renováveis, as quais, já sinalizam indícios de escassez para as próximas décadas [1][2]. Estes e outros fatores têm influenciado o aumento da taxa média de crescimento mundial de biocompósito que entre 2003 e 2007 foi de $38 \%$ ao ano, nesse mesmo período, a taxa de crescimento anual na Europa foi de $48 \%$. Há, no entento, uma

ITEGAM - JETIA Vol. 01, №.04. Dezembro de 2015. Manaus - Amazonas, Brasil. ISSN 2447-0228 (ONLINE). 
previsão de superação da capacidade de produção que era de 0,36 milhões de tonelada ocorrida no ano de 2007, para um aumento de 2,33 milhões de toneladas até 2013, e 3,45 milhoes de toneladas até 2020 [3][4].

Este cenário tem contribuído para uma maior participação de fibras provenientes de fontes renováveis na indútria devido: sua baixa densidade, baixo custo, estimula a geração de renda no campo, possuem boas propriedades mecânicas, físicas e químicas, amplamente distribuídas, baixo consumo de energia na produção e no processamento, disponíveis, moldáveis, maciez e abrasão reduzida, porosas, viscoelásticas, biodegradáveis, promove um processo seguro sem risco de contaminação do trabalhador, entre outros [3][5].

Diante dessa perspectiva, no Brasil, a região Amazônica por ser uma região de clima tropical, possui uma diversidade de plantas propicias ao desenvolvimento de novos compósitos, como é o caso da fibra de Munguba (Pseudobombax munguba) e Jitó (Guarea guidonia). Estas, junto com fibras de outras espécies de plantas são utilizadas no cotidiano por moradores da região amazônica na fabricação de cordas envira, usadas na confecção de cestos, redes, utensílios de pesca e de caça, objetos artesanais e outros. Podendo estas ser mais uma alternativa ao desenvolvimento de novos compósitos poliméricos.

\section{METODOLOGIA APLICADA}

Resina Poliéster: a resina utilizada foi a poliéster tereftálica insaturada e pré-acelerada, fabricada pela Royal Polímeros sob a denominação comercial de Denverpoly 754. $\mathrm{O}$ agente de cura utilizado foi o peróxido de MEK (Butanox M-50), na proporção de $0,33 \%$ (v/v). A resina foi adquirida já pré-acelerada com naftenato de cobalto (CoNap) na proporção mássica de $0,15 \%$. A proporção de agente de cura/resina de $0,33 \%(\mathrm{v} / \mathrm{v})$, utilizada tende a melhorar o desempenho mecânico do compósito [6][7][8].

Fibra de Munguba e Jitó: o desfibramento e a separação das fibras foram realizados manualmente, em seguida, cortadas com estiletes e tesouras manuais em que se obtiveram fibras nos comprimentos de $10 \mathrm{~mm}$ e $15 \mathrm{~mm}$, sendo este um procedimento consideravelmente simples, uma vez que as fibras já haviam permanecido submersas em água em um recipiente por 48 horas, cujo intuito era torna-las maleáveis e de fácil manuseio.

Confecção dos copos de prova: a confecção dos corpos de prova dos compósitos consistiu em um processo simplificado, com o uso de moldagem manual, utilizando moldes de silicone sem o uso de desmoldante e sem pressão, utilizando como matriz poliéster reforçada por fibras curtas dispostas aleatoriamente nos comprimentos de 10 e $15 \mathrm{~mm}$, conforme proporções mássicas para os compósitos com fibras de Munguba (10,58\% e 5,22\%) e os com fibras de Jitó (10mm $-10,58 \%$ e $15 \mathrm{~mm}-5.22 \% \%)$.
Ensaios de tração do material compósito: Os ensaios de tração ocorreram em conformidade com a norma ASTM D 638M. A confecção dos compósitos para o ensaio de tração ocorreu mediante o uso de moldes de silicone com um número de 24 (vinte e quatro) corpos de prova com matriz reforçada por fibras curtas de Munguba e de Jitó. Os quais foram ensaiados em uma máquina universal modelo KRATOS MKCA-KE, com sistema de aquisição de dados, com célula de carga de $5 \mathrm{KN}$, adotando velocidade de 5 $\mathrm{mm} / \mathrm{min}$ e comprimento útil para medição entre garras de 60 $\mathrm{mm}$.

Análises da superfície da fratura dos corpos de prova: A análise da superfície da fratura dos compósitos ocorreu mediante Microscopia Eletrônica de Varredura - MEV, em que se analisou a morfológica da superfície da fratura e os principais mecanismos de falha de cada compósito ensaiado.

\section{III.RESULTADOS E DISCUSSÕES}

Caracterização das fibras de Munguba e Jitó: as fibras de Munguba e Jitó in natura foram caracterizadas quanto ao comprimento $(\mathrm{mm})$, determinação da massa especifica (\%) e aspectos superficiais. A análise microestrutural ocorreu mediante Microscopia Eletrônica de Varredura-MEV, em que se verificou o aspecto superficial e a seção transversal das fibras, além de amostras embutidas na matriz de poliéster tereftálico.

O valor médio da massa especifica das fibras de Munguba e de Jitó ocorreu em concomitância ao comprimento médio das fibras, sendo estas pesadas em balança de precisão modelo Marte, da Marte Balança e Aparelhos de Precisão LTDA. A determinação do teor de umidade/absorção máxima das fibras se deu mediante a secagem destas num período de 15 minutos em forno elétrico modelo Layr Midy Inox, onde se determinou o teor base de umidade em três (3) amostras de fibras, com massa total de 34,50 gramas. As fibras foram inicialmente pesadas e secas até a massa constante.

Características mecânicas e microestrutural dos materiais compósitos: a fabricação, acabamento, dimensionamento e marcação dos compósitos com fibra de Munguba e de Jitó ocorreram conformidade com especificações das normas ASTM D 638.

Materiais compósitos com fibras de Munguba e de Jitó de $10 \mathrm{~mm}$ e $15 \mathrm{~mm}$ : A Tabela 1 mostra os resultados obtidos no ensaio mecânico de tração para os compósitos de poliéster reforçados por fibras de Munguba e de Jitó nos comprimentos de $10 \mathrm{~mm}$. 
Raimundo V. P. Lopes e Eleodoro Rodriguez Hermenegildo/ITEGAM-JETIA Vol.01, No 04. pp. 04-08. Dezembro, 2015.

Tabela 1. Resultado do ensaio de tração de compósitos poliéster tereftálico reforçados por fibras de Munguba e de Jitó nas proporções de $10 \mathrm{~mm}$.

\begin{tabular}{|c|c|c|c|c|c|}
\hline Compósitos & $\begin{array}{c}\text { Força } \\
(\mathrm{kN})\end{array}$ & $\begin{array}{c}\text { Deslocamento } \\
(\mathrm{mm})\end{array}$ & $\begin{array}{c}\text { Tensão Max } \\
(\mathrm{Mpa})\end{array}$ & $\begin{array}{c}\text { Módulo de Elasticidade } \\
(\mathrm{GPa})\end{array}$ & $\begin{array}{c}\text { Deformação } \\
(\mathrm{mm} / \mathrm{mm})\end{array}$ \\
\hline Munguba & 0,40 & 2,69 & 12,16 & 0,27 & 0,04 \\
\hline Jitó & 0,91 & 2,36 & 18,84 & 0,48 & 0,04 \\
\hline
\end{tabular}

A Figura 1 mostra o gráfico Tensão $(\mathrm{MPa})$ versus Deformação (mm) dos corpos de prova após o ensaio de tração

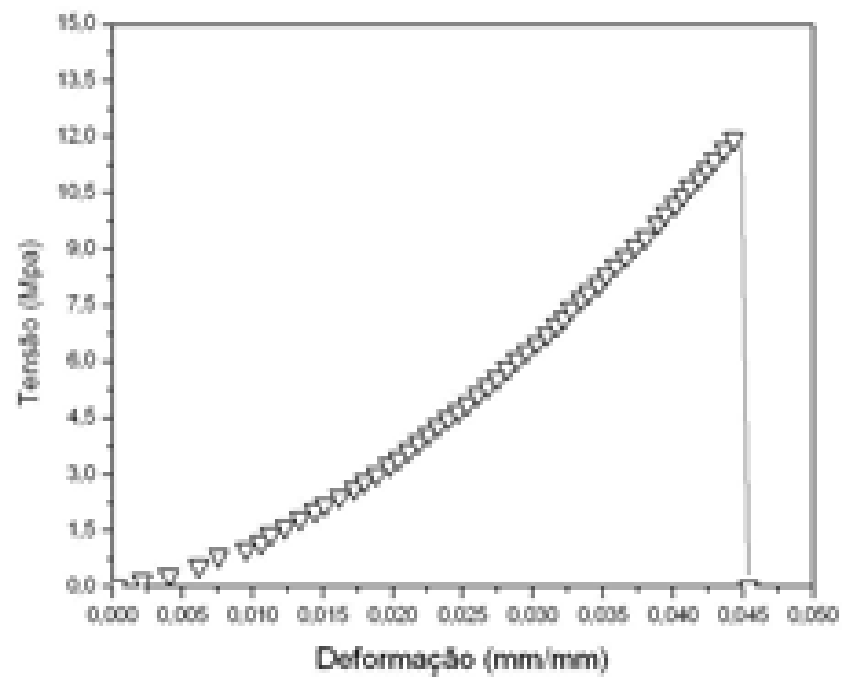

para compósito de poliéster reforçado por fibras de munguba e de Jitó de $10 \mathrm{~mm}$ de comprimento, em que se obteve uma diferença de $21 \%$ (MPa), em comparação aos com fibras de Jitó de $10 \mathrm{~mm}$.

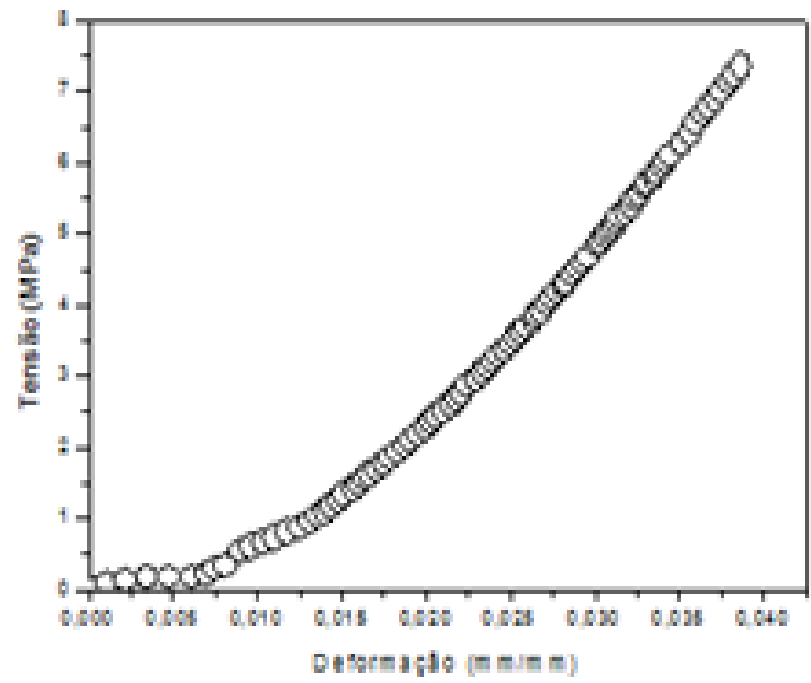

Figura 1: Tensão (MPa) versus Deformação $(\mathrm{mm} / \mathrm{mm})$ de corpos de prova de com fibras de $10 \mathrm{~mm}$.

$\mathrm{Na}$ análise interna da fratura de compósito com fibras de Munguba de $10 \mathrm{~mm}$ por meio de MEV foi constatado uma maior resistência mecânica dos compósitos, considerando a integridade das fibras e da superfície do mesmo, em comparação aos com fibras de $5 \mathrm{~mm}$. Constata-se ainda,ocorrência de arrancamento (pull out) de fibras e
Materiais compósitos com fibras de Munguba e de Jitó de $15 \mathrm{~mm}$ : A Tabela 2 apresenta os resultados obtidos nos ensaios de resistência à tração mecânica dos compósitos reforçados por fibras de $15 \mathrm{~mm}$. Fornecendo uma melhor compreensão do esforço mecânico aplicado e suas proporcionalidades nos compósitos, em que se obteve uma diferença de $52 \%$ (MPa) em comparação com os reforçados por fibras de $5 \mathrm{~mm} \mathrm{e}, 39 \%$ (MPa) em comparação com os reforçados por fibras de $10 \mathrm{~mm}$.

Tabela 2. Resultados dos ensaios de tração dos compósitos de poliéster tereftálico reforçado por fibras de Munguba e de Jitó nas proporções de $15 \mathrm{~mm}$.

\begin{tabular}{|c|c|c|c|c|c|}
\hline Compósitos & $\begin{array}{c}\text { Força } \\
(\mathrm{kN})\end{array}$ & $\begin{array}{c}\text { Deslocamento } \\
(\mathrm{mm})\end{array}$ & $\begin{array}{c}\text { Tensão Max } \\
(\mathrm{Mpa})\end{array}$ & $\begin{array}{c}\text { Módulo de Elasticidade } \\
(\mathrm{GPa})\end{array}$ & $\begin{array}{c}\text { Deformação } \\
(\mathrm{mm} / \mathrm{mm})\end{array}$ \\
\hline Munguba & 0,63 & 3,03 & 19,91 & 0,39 & 0,05 \\
\hline Jitó & 1,07 & 1,95 & 19,08 & 0,60 & 0,03 \\
\hline
\end{tabular}


A Figura 2 mostra o gráfico Tensão (MPa) versus Deformação $(\mathrm{mm})$ dos corpos de prova após o ensaio de tração para o compósito de Poliéster reforçado por fibras de munguba e de Jitó de 15 mm de comprimento.

$\mathrm{Na}$ caracterização microetrutural interna do compósito com fibras de $15 \mathrm{~mm}$ foi evidenciado uma drástica redução de crateras e arrancamento de fibras (pull out) na matriz, com ocorrência de trincas (resultado da concentração de tensões) com as fibras se mantendo integras durante esforço mecânico aplicado. Tal fato foi influenciado pelo comprimento das fibras, uma vez que estas ocupavam uma área de contato maior na matriz, além de estarem melhor alinhadas e uniformes, o que promoveu, por consequência, melhor interação interfacial fibramatriz facilitando a transferência de carga da matriz para as fibras, garantindo dessa forma, resultados mecânicos mais satisfatórios para este tipo de compósito [9].

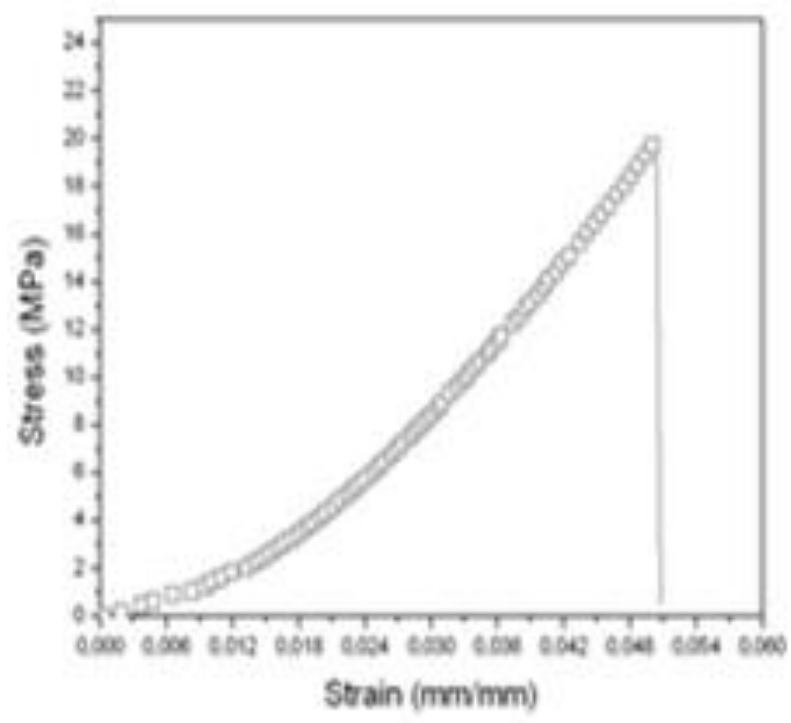

Conforme a determinação do comprimento ( $\mathrm{mm})$, massa especifica (\%) e aspectos superficiais das fibras nas propriedades do compósito com resina poliéster tereftalico, reforçado por fibras curtas de mungubas e de Jitó dispostas aleatoriamente, os resultados indicaram que: $\mathrm{O}$ aumento nas propriedades mecânicas para ambos os compósitos no ensaio de resistência a tração foram respectivos aos comprimentos das fibras e, a medida que estas se mantinham melhor alinhadas, uniformes e passavam a ocupar uma área de contato maior na matriz conforme MEV. Promovendo melhor transferência de carga da matriz para as fibras.

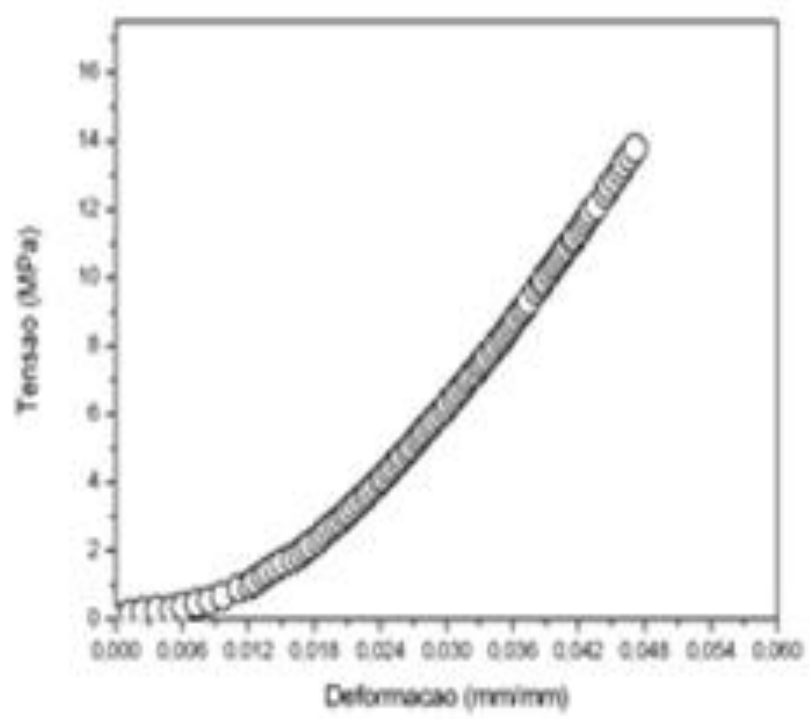

Figura 2: Força $(\mathrm{N})$ versus alongamento $(\mathrm{mm})$ de compósito com fibras de munguba (esquerda) e Jitó (direita) de $15 \mathrm{~mm}$.

\section{CONCLUSÃO}

A fabricação de compósitos de poliéster reforçados por fibras amazônicas de Munguba e Jitó ocorreu mediante um processo consideravelmente simples, com um menor nível possível de processamento nas etapas produtivas, em que se obteve um material tecnicamente viável do ponto de vista da engenharia dos materiais; podendo inferir diante do exposto que, os compósitos em questão possuem propriedade mecânica adequadas no contexto do uso e desenvolvimento de materiais compósitos poliméricos. Ao mesmo tempo contribui com as discursões em torno da viabilidade no uso de fibras vegetais amazônicas, para o desenvolvimento de novos compósitos polimérico, em prol da pesquisa cientifica e da sustentabilidade.

\section{AGRADECIMENTOS}

Fundação de Amparo à Pesquisa do Estado do Amazonas (FAPEAM) e ao Instituto de natureza e Cultura/INC/UFAM/BC pelo apoio na realização dessa pesquisa a Universidade Federal do Amazonas (UFAM) e a Universidade Federal do Pará (UFPA). 


\section{REFERÊNCIAS BIBLIOGRÁFICAS}

[1] Monteiro, S. N.; Calado, V.; Margem, F. M.; Rodriguez, R. J. S. Thermogravimetric Stability Behavior of Less Common Lignocellulosic Fibers. 2012, v.1, 3, 189-199.

[2] Liu, H. et al, Compatibilizing and toughening bamboo flour-filled HDPE composites: Mechanical properties and morphologies. Composites: Part A. 2008, n. 39, p. 1891-1900.

[3] Faruk, O.; Bledzki, A. K.; Fink, H.; Sain, M. Biocomposites reinforced with natural fibers: $\mathbf{2 0 0 0}$ - 2010. 2012. 37, 15521596.

[4] Mothé, C. G. and ARAÚJO, C. R., Caracterização térmica e mecânica de compósitos de poliuretano com fibras de Curauá. Polímeros: Ciência e Tecnologia. 2004, v. 14, n. 4, p.274-278.

[5] Levy Neto, F. L.; Pardini, L. C. Compósitos Estruturais: Ciência e Tecnologia. Ed. Edgard, São Paulo, 2006.

[6] Rodrigues, J. S., Análise fractográfica de compósitos de matriz poliéster e resíduos da indústria madeireira. VI Congresso Nacional de Engenharia Mecânica - CONEM, 18 a 21 de Agost. 2010, Campina Grande/PB.

[7] Khanam, P. N. et al, Tensile, Flexural and Compressive Properties of Sisal/Silk Hybrid Composites. Journal of Reinforced Plastics and Composites. 2007, v. 26, n. 10.

[8] Venkateshwaran, N. and Elayaperumal, A. Modeling and evaluation of tensile properties of randomly oriented banana/epoxy composite. Journal of Reinforced Plastics and Compósite. 2011, n. 30, p. 1957-1967.

[9] Harnnecker, F. et al. Biodegradable Polyester-Based Blend Reinforçed with Curauá Fiber: Thermal, Mechanical and Biodegradation Behaviour. Journal of Polymers and the Environment. 2012, vol. 20, p. 237-244. 